\title{
Repertorio léxico, campos semánticos y relaciones semánticas de tipo jerarquización de significados en niños peruanos de 5 años
}

\author{
Lexical Repertoire, Semantic Fields and Semantic \\ Relations of Hierarchization type of Meanings in \\ Five Years old Peruvians Children
}

\section{Sandra Manrique C.} Psicóloga

Magíster en Educación con Mención en Trastornos de la Comunicación Humana Departamento de Investigación, Capacitación y Proyectos Especiales del Centro Peruano de Audición, Lenguaje y Aprendizaje, CPAL, Perú

\footnotetext{
Contacto con el autor: Sandra Manrique $\mathrm{C}$. Martín Pizarro 172 Valle Hermoso - Surco Lima - Perú Tel: (00 51 1) 7069081 Correo-e: smanrique@cpal.edu.pe

Recibido: 27/04/2015 Aceptado: 01/10/2015
}

\begin{abstract}
RESUMEN
El objetivo del estudio fue determinar las características del repertorio léxico, campos semánticos y relaciones semánticas de tipo jerarquización de significados, en niños peruanos de 5 años. Se evaluó a 1.564 estudiantes de instituciones de educación básica regular de gestión estatal ubicadas en las ciudades de mayor densidad demográfica de las zonas norte, centro y sur del Perú. El instrumento empleado fue el Sub teste vocabulário del Teste de Linguagem Infantil nas áreas de fonologia, vocabulário, fluência e pragmática $A B F W$. Los resultados obtenidos en el nivel de repertorio léxico y relaciones semánticas de inclusión de tipo jerarquización de significados indicaron que la edad influye en el desempeño de los examinados. En el nivel de campos semánticos, el género y la edad influyen en el rendimiento.
\end{abstract}

Palabras clave: repertorio léxico, campos semánticos, relaciones semánticas de inclusión de tipo jerarquización de significados.

\section{ABSTRACT}

This study aims to determine the characteristics of semantic performance in aspects related to lexical repertoire, semantic fields and semantic relations in hierarchy type of meanings in 1.564 5-year-old Spanish speaking Peruvian children. The participants did not have special education needs. They belonged to regular public school, located in the most densely populated cities of northern, central and southern Peru. The Sub teste vocabulário of Test de Linguagem Infantil nas áreas de fonología, vocabulário, fluência e pragmática $A B F W$ was administered. The results about lexical repertoire and inclusion semantic relations of the type of meaning hierarchy showed that gender did not influence the performance of the participants. It was also observed that age and region of residence have an influence on these aspects. Furthermore, gender, age and region of residence affected the performance of the participants in semantic fields.

Keywords: lexical reperttoire, semantic fields, semantic relations of hierarchization type of meanings. 


\section{Introducción}

La semántica es la dimensión que abarca el contenido del lenguaje e implica el estudio del significado de las palabras y sus combinaciones (Acosta, Moreno, Ramos, Quintana y Espino, 2002). Los estudios acerca de la adquisición y desarrollo del lenguaje resaltan la importancia de la semántica. Así, Bronckart (citado por Acosta et al., 2002) señala que la realidad de una lengua existe a través del contenido que se transmite $y$ es aprendida inicialmente por medio de aspectos semánticos y pragmáticos antes de centrarse en los aspectos morfológicos y sintácticos. En este sentido, el desarrollo semántico se considera un importante índice del lingüístico (Mayor, Zubiauz y Díez-Villoria, 2005) y, por ello, genera en la actualidad un gran interés en sí mismo.

La semántica, desde una perspectiva aplicada (Acosta y Moreno, 2005), incluye el análisis del léxico y del proceso de significación, ya que en el ámbito infantil es de gran interés tanto la adquisición y crecimiento del sistema léxico (competencia léxica), como el desarrollo conceptual (competencia semántica).

El léxico, por su parte, es un sistema de unidades mínimas que poseen significado y están relacionadas entre sí por reglas que permiten y controlan la creación de nuevas palabras (Rojas, 2011). En relación con el desarrollo del léxico, la literatura señala que existen diferencias individuales en la adquisición del repertorio inicial (Galián, Carranza y Escudero, 2006). Desde esta perspectiva, los niños adquirirían el lenguaje a ritmos y estilos individuales de desarrollo, los que varían considerablemente, sobre todo respecto del aprendizaje de las palabras
(Galián, Ato y Carranza, 2010). Así mismo, autores como Dale (1989) y Karmiloff y Karmiloff-Smith (2005) señalan que existen notables variaciones en cuanto al tamaño y contenido del léxico infantil, no solo entre un niño y otro, sino también entre niños y niñas. Huttenlocher (citado por Galián et al., 2006) sostiene que las diferencias en función al sexo se observan en especial en la etapa de adquisición de las primeras 50 palabras, desapareciendo aproximadamente a partir de los 24 meses. En este sentido, Trigo (citado por Ruiz, 2000) en un estudio en 360 niños de 5 a 11 años de Sevilla, encontró que en el desarrollo del lenguaje, la variable edad es un factor que genera mayor diferencia que la variable sexo.

Por otra parte, para Acosta y Moreno (2005) los campos semánticos, llamados también "categorías", son agrupaciones de distintos elementos que tienen características o rasgos comunes o "semas" (unidad mínima de significación en la que una palabra se puede descomponer), que identifican o diferencian a la vez los elementos de ese grupo de los demás (Martínez, 2002). En relación con la adquisición y desarrollo de los diferentes elementos que forman los campos semánticos, Owens (citado por Gallego, 2006) señala que las primeras palabras infantiles suelen referirse a nombres de juguetes, alimentos y animales; además de los referentes familiares típicos.

Gallego (2006), en tanto, señala que hacia los 4 años, luego de un largo camino en la adquisición del léxico, el niño comienza a tener un cierto dominio de las relaciones semánticas que se establecen entre las palabras y hace también un uso más apropiado del léxico de su lengua. 
Al mismo tiempo, incrementa considerablemente su capacidad para entender los mensajes y para darse cuenta de algunos mensajes implícitos en la conversación, así como también se incrementa de modo significativo el léxico, lo cual le facilita el uso de ciertas estrategias para su mejor organización. En este momento, el aprendizaje de las relaciones de significado ocupa un lugar importante ya que, como se sabe, es fundamental para lograr la competencia semántica (Dale, 1989).

Al respecto, existen diferentes propuestas para clasificar las relaciones entre los significados de las palabras. Navarro (2006), por ejemplo, considera la identidad de significados, la identidad de significantes, la oposición de significados y la jerarquización de significados. En esta última se presentan los siguientes fenómenos (Moreno, 2008).

- Hiperónimo. Término cuyo significado incluye el de otro. Su significado es más extenso que el de los hipónimos. Se le denomina "conjunto/clase incluyente".

- Hipónimo. Consiste en una relación de inclusión de un significado respecto de otro, es decir, es un término cuyo significado se incluye en otro más amplio. Se le denomina "conjunto/clase incluida".

- Cohipónimo. Corresponde a dos o más palabras que son hipónimos de un mismo hiperónimo.

Por otra parte, los enfoques relacionados con el estudio de la semántica abordan dos aspectos: el primero pone énfasis en los aspectos cognitivos para explicar el desarrollo semántico del niño y el segundo considera la profunda influencia de los estímulos externos. Dentro de esta última perspectiva, más dinámica e interactiva para explicar el desarrollo del lenguaje, autores como Rondal (1983), Bruner (1983) y Del Río (1985) (citados por Acosta et al., 2002) expresan la importancia que juega el contexto y las variables socioafectivas. Desde esta perspectiva, el desarrollo semántico del niño se ve afectado por la calidad de sus interacciones con el medio y por la manera en la que utiliza el lenguaje que lo rodea. En última instancia, las variables de tipo familiar, socioeconómicas y educativas deben ser contempladas.

Considerando la importancia de la semántica en el desarrollo lingüístico y evidenciándose escasos estudios acerca del desarrollo del repertorio léxico, campos semánticos y relaciones semánticas de tipo jerarquización de significados en niños peruanos, el presente estudio pretende contribuir en esta área del conocimiento planteándose la siguiente interrogante: ¿Cuáles son las características del repertorio léxico, campos semánticos y relaciones semánticas de tipo jerarquización de significados en niños peruanos según sexo y rango de edad?

\section{Objetivos}

Los objetivos del estudio son los siguientes: a) Determinar las características de presentación del repertorio léxico según sexo y rango de edad en niños peruanos de 5 años; b) Describir las características de presentación de los campos semánticos según sexo y rango de edad en niños peruanos de 5 años; y c) Describir las características de presentación de las relaciones semánticas de tipo jerarquización de significados según sexo y rango de edad en niños peruanos de 5 años. 


\section{Método}

El estudio es de tipo cuantitativo, de alcance descriptivo y el diseño empleado es transversal y descriptivo comparativo (Hernández, Fernández y Baptista, 2006).

\section{Participantes}

La muestra estuvo conformada por 1.564 niños (Tabla 1), obtenidos a través de un muestreo de tipo no probabilístico en su modalidad por cuotas de afijación uniforme, es decir, se procuró que en cada región participara un mismo número de niños.

Los criterios de inclusión en la muestra fueron los siguientes: niños y niñas de 5 años de edad (que para el análisis de resultados se dividieron en tres rangos: 5 años 3 meses a 5 años 5 meses; 5 años 6 meses a 5 años 8 meses; y 5 años 9 meses a 5 años 11 meses) que cursan el nivel inicial en instituciones educativas estatales de las ciudades o distritos más poblados de regiones distribuidas en las zonas norte (Cajamarca, Piura, La Libertad y San Martín), centro (Lima Provincias y Lima Metropolitana) y sur (Puno, Arequipa, Madre de Dios, Ayacucho y Huancavelica) del Perú; de lengua materna español y $\sin$ necesidades educativas especiales según el reporte de las fichas de inscripción de las instituciones educativas.

Tabla 1

Composición de la muestra

\begin{tabular}{|c|c|c|c|c|}
\hline \multirow{2}{*}{ Zonas } & \multirow{2}{*}{ Ciudad } & \multicolumn{2}{|c|}{ Sexo } & \multirow{2}{*}{ Total } \\
\hline & & Mujeres & Varones & \\
\hline \multirow{4}{*}{ Norte } & Cajamarca & 85 & 69 & 154 \\
\hline & Piura & 71 & 81 & 152 \\
\hline & La Libertad & 90 & 69 & 159 \\
\hline & San Martin & 66 & 69 & 135 \\
\hline \multirow{5}{*}{ Sur } & Puno & 72 & 50 & 122 \\
\hline & Arequipa & 76 & 78 & 154 \\
\hline & Madre de Dios & 75 & 69 & 144 \\
\hline & Ayacucho & 71 & 83 & 154 \\
\hline & Huancavelica & 49 & 54 & 103 \\
\hline \multirow{2}{*}{ Centro } & Lima Provincias & 74 & 62 & 136 \\
\hline & Lima Metropolitana & 76 & 75 & 151 \\
\hline & Total & 805 & 759 & 1.564 \\
\hline
\end{tabular}

\section{Procedimientos}

Se coordinó con los Gobiernos Regionales y se obtuvieron los permisos de las 22 instituciones comprometidas, contando para esta tarea con el apoyo del Consejo Nacional de Educación del Perú. Las regiones tuvieron a su cargo la selección de los centros educativos para garantizar la evaluación de aproximadamente 150 niños por región y la coordinación de la actividad con los directores (finalidad, fecha, horario, ambientes, número y asistencia de niños, personal de apoyo, envío de las fichas de matrícula de las aulas de cinco años con anticipación, número de salones u oficinas, mobiliario, electricidad y lugares silenciosos).

Por otro lado, anticipadamente se contactaron especialistas, psicólogos, docentes y estudiantes de los últimos años de Psicología y Educación, quienes fueron capacitados en la administración del test. 
En cada visita a las ciudades, el equipo de evaluadores fue responsable de la administración de las pruebas, así como de los recursos materiales y humanos.

La administración de la prueba se realizó de forma individual, sin considerar un tiempo límite de aplicación, en los ambientes de las instituciones educativas convocadas, siguiendo las recomendaciones pertinentes del caso: iluminación adecuada y ausencia de estímulos sonoros que afectaran el desempeño de los niños.

\section{Instrumento}

Se utilizó el Subteste vocabulário del Teste de Linguagem Infantil nas áreas de fonologia, vocabulário, fluência e pragmática ABFW (Furquim de Andrade, Befi-Lopes, Dreux y Fiszbein, 2004) Versión adaptada.

La prueba consiste en mostrar las imágenes de cada uno de los campos semánticos en el siguiente orden: vestuario, animales, alimentos, medios de transporte, muebles y utensilios, profesiones, lugares, formas y colores, juguetes e instrumentos musicales. Si el niño no denomina la figura en 10 segundos o dice que no sabe, se debe pasar a la siguiente imagen, repitiendo la pregunta correspondiente. Antes de iniciar la evaluación del siguiente campo semántico se le deben presentar nuevamente las figuras que no logró denominar, respetando el orden numérico. El test permite realizar dos tipos de análisis:

- Análisis cuantitativo para obtener el repertorio léxico por campos semánticos. Se corrigen las respuestas del niño y se obtiene el número de elementos para cada una de las categorías: DVU (designación por vocablo usual), ND (no designación) y PS (procesos de sustitución). De este modo se llena el cuadro de resultados obtenidos y se contrasta con los resultados esperados según el rango de edad.

A través de la sumatoria de respuestas correctas (DVU) se obtiene el repertorio léxico en cada campo semántico, lo que permitirá posteriormente realizar la comparación de los campos semánticos entre sí, con el objetivo de determinar cuáles son los menos conocidos y los más conocidos, por el niño.

- Análisis cualitativo. Con los resultados obtenidos en PS (procesos de sustitución), se realiza el análisis de las sustituciones realizadas por los niños, lo cual permite indicar las relaciones semánticas usadas por el niño. En la prueba original consideran los siguientes tipos de sustitución: parasinónimos, hiperónimos, hipónimos y cohipónimos. En el presente estudio se analizará solo la presencia de hiperónimos, hipónimos y cohipónimos, lo cual se vincula con las relaciones semánticas de tipo jerarquización de significados que son objeto de la investigación.

Adaptación del Subteste vocabulário del Teste de Linguagem Infantil nas áreas de fonologia, vocabulário, fluência e pragmática ABFW

Parte del presente estudio implicó la adaptación del subtest de vocabulario que consistió en la traducción del manual y del protocolo de respuesta a cargo de tres especialistas en trastornos del lenguaje infantil. Posteriormente se realizó el estudio psicométrico para precisar las características de confiabilidad y validez, así como las normas para la interpretación de los resultados. 
El estudio psicométrico consideró el análisis de los ítems con el objetivo de determinar los reactivos que contribuyen a la validez y confiabilidad desde un punto de vista intrínseco de la prueba. Para tal efecto se calculó el índice de discriminación de cada reactivo y el coeficiente de consistencia interna de Cronbach. Los reactivos que no alcanzaron un índice de discriminación igual o menor a .20 fueron desechados, quedando 68 reactivos, los cuales conforman la versión adaptada del Subteste de vocabulário del Teste de Linguagem nas áreas de fonologia, vocabulário, fluência e pragmática ABFW.

Se calculó el coeficiente de confiabilidad de las puntuaciones obtenidas, utilizando el método de consistencia interna. El valor del coeficiente Alfa de Cronbach para la versión adaptada fue de 0.89 , por lo cual la prueba es dimensionalmente consistente y fiable.

En cuanto a la validez, si bien las correlaciones son estadísticamente muy significativas, sus grados de asociación no alcanzaron el valor de 0.70 , lo que indicaría colinealidad, por lo que puede considerarse que teniendo un núcleo común, los campos conceptuales son diferentes. Estos resultados indican

Tabla 2

Análisis inferencial de diferencias en el repertorio léxico según sexo

\begin{tabular}{cccccc}
\hline Sexo & $n$ & $M$ & $D E$ & $t$ & $P$ \\
\hline Femenino & 805 & 38.43 & 9.85 & \multirow{2}{*}{1.72} & \multirow{2}{*}{085} \\
Masculino & 759 & 39.35 & 9.79 & & \\
\hline
\end{tabular}

$n=1.564$

En la Tabla 3 se observa que en el repertorio léxico, según los tres rangos para la edad de 5 años establecidos para la comparación, existen diferencias estadísticamente significativas en los tres grupos $(F=$ 20.39, $p<.000)$. El análisis post hoc realizado con la prueba de Bonferroni evidencia que el grupo de 5 años 3 meses a 5 años 5 meses difiere que la prueba de vocabulario tiene visos de validez, a lo cual contribuye también el valor de los índices de discriminación de los ítems.

En síntesis, la prueba es confiable y válida para el logro de sus propósitos.

\section{Resultados}

Para el logro de los objetivos se analizaron los datos recolectados a través de estadísticos descriptivos (Media y Desviación estándar) e inferenciales ( $t$ de Student para muestras independientes, ANOVA y la prueba post hoc de Bonferroni) al nivel de significación del .05 a través del software estadístico SPSS (Statistical Package for Social Science) versión 17.

A continuación se presentan los resultados del estudio considerando los objetivos planteados: repertorio léxico, campos semánticos y relaciones semánticas de tipo jerarquización de significados.

\section{Repertorio léxico}

La Tabla 2 muestra que en el nivel de repertorio léxico de los grupos no se diferencian significativamente $(t=1.72, p>.05)$. Sin embargo, el promedio es mayor en los niños que en las niñas. significativamente tanto del grupo de 5 años 6 meses a 5 años 8 meses como del grupo de 5 años 9 meses a 5 años 11 meses. En ambos casos el grupo de menor rango de edad es el que obtuvo menores puntuaciones promedio. No se observan diferencias entre los dos grupos de mayor edad $(p>.01)$. 
Tabla 3

Análisis inferencial de diferencias en el repertorio léxico entre los grupos etários de 5.3 - 5.5 años, 5.6 - 5.5 años y 5.9 - 5.11 años

\begin{tabular}{cccccc}
\hline Grupos de edad & $n$ & $M$ & $D E$ & $F$ & \\
\hline $5.3-5.5$ años & 483 & 36.72 & 10.15 & & \\
$5.6-5.8$ años & 510 & 39.52 & 9.41 & 20.39 & .0000 \\
$5.9-5.11$ años & 571 & 40.83 & 9.44 & & \\
\hline$n=1.546$ & & &
\end{tabular}

\section{Campo semántico}

Respecto de las características de los campos semánticos a nivel global, a partir de la contrastación de la media teórica (valor de prueba .50) con la media de los puntajes alcanzados por la muestra, se encontró que existen diferencias significativas ( $p<$
.05) en los campos semánticos animales, alimentos, muebles y utensilios, profesiones, lugares, formas y colores y juguetes e instrumentos musicales, a excepción de los campos vestuario $(t=4.46, p>.05)$ y medios de transporte $(t=4.18, p>.05$ ) (ver Tabla 4).

Tabla 4

Análisis inferencial de diferencias entre la media teórica de la prueba y la media de los puntajes alcanzados por la muestra en función del campo semántico

\begin{tabular}{lllccc}
\hline & & & & \multicolumn{3}{c}{ Valor de prueba $=.50$} \\
\cline { 3 - 6 } Campos semánticos & $M$ & $D E$ & $t$ & $\begin{array}{c}\text { Diferencia de } \\
\text { medias }\end{array}$ \\
\hline Vestuario & .50 & .31 & 4.46 & .00 & .645 \\
Animales & .73 & .27 & 6.81 & .23 & .000 \\
Alimentos & .61 & .17 & 5.64 & .12 & .000 \\
Medios de transporte & .49 & .24 & 4.18 & -.01 & .215 \\
Muebles y utensilios & .60 & .18 & 5.56 & .11 & .000 \\
Profesiones & .38 & .20 & 2.78 & -.12 & .000 \\
Lugares & .35 & .21 & 2.63 & -.14 & .000 \\
Formas y colores & .85 & .18 & 8.07 & .35 & .000 \\
Juguetes e instrumentos musicales & .54 & .21 & 4.87 & .05 & .000 \\
\hline
\end{tabular}
$n=1.564$

En la Tabla 5 se evidencia, a partir del análisis de las características de los campos semánticos según sexo, que existen diferencias estadísticamente significativas en los campos semánticos vestuario ( $t=$ 10.12, $p<.05)$, a favor del sexo femenino y en los campos semánticos animales $(t=-3.07, p<.05)$, medios de transporte $(t=-10-25, p<.05)$, lugares $(t$ $=-2-25, p<.05)$ y juguetes e instrumentos musicales $(t=-4.08, p<.05)$, a favor del sexo masculino. 
Tabla 5

Diferencia de medias del desempeño en cada campo semántico en función al sexo

\begin{tabular}{|c|c|c|c|c|c|c|}
\hline \multirow{2}{*}{ Campos semánticos } & \multicolumn{2}{|c|}{ Femenino } & \multicolumn{2}{|c|}{ Masculino } & \multirow{2}{*}{$t$} & \multirow{2}{*}{$P$} \\
\hline & $M$ & $D E$ & $M$ & $D E$ & & \\
\hline Vestuario & .58 & .30 & .42 & .30 & 10.12 & .000 \\
\hline Animales & .71 & .28 & .76 & .27 & -3.07 & .002 \\
\hline Alimentos & .62 & .17 & .61 & .17 & 1.05 & .295 \\
\hline Medios de transporte & .43 & .24 & .56 & .23 & -10.25 & .000 \\
\hline Muebles y utensilios & .61 & .18 & .61 & .18 & -.224 & .823 \\
\hline Profesiones & .38 & .21 & .39 & .20 & -097 & .330 \\
\hline Lugares & .35 & .21 & .37 & .22 & -2.25 & .025 \\
\hline Formas y colores & .89 & .17 & .85 & .19 & .68 & .493 \\
\hline Juguetes e instrumentos musicales & .53 & .21 & .57 & .21 & -4.08 & .000 \\
\hline
\end{tabular}

$n=1.564$

Como se puede apreciar en la Tabla 6, existen diferencias estadísticamente significativas en función de la edad en todos los campos semánticos (animales, alimentos, medios de transporte, muebles y utensilios, profesiones, lugares, formas y colores, juguetes e instrumentos musicales) $(p<.05)$, con excepción de vestuario $(F=1.99, p>.05)$.

A partir del análisis post hoc mediante la prueba de Bonferroni se encontró que en los campos semánticos animales y lugares, solo existen diferencias significativas $(p<.01)$ entre el grupo de edad de 5 años 9 meses a 5 años 11 meses y el grupo de edad de 5 años 3 meses a 5 años 5 meses, a favor del primer grupo en ambos casos. En los campos semánticos: alimentos, transportes, muebles, profesiones y juguetes e instrumentos, además de existir diferencias significativas entre los grupos de edad de 5 años 9 meses a 5 años 11 meses y de 5 años 3 meses a 5 años 5 meses, a favor de los primeros, también existen diferencias significativas $(p<.01)$ entre los grupos de edad de 5 años 6 meses a 5 años 8 meses y el grupo de 5 años 3 meses a 5 años 5 meses, también a favor del primer grupo. Por último, en el campo semántico formas y colores existen diferencias significativas $(p<.01)$ entre cada par de grupo de edad, siempre a favor de los grupos de mayor rango de edad.

Tabla 6

Análisis de varianza del repertorio léxico en cada campo semántico entre los grupos etários de 5.3 - 5.5 años, 5.6 - 5.8 años y 5.9 -5.11 años

\begin{tabular}{|c|c|c|c|c|c|c|c|c|}
\hline \multirow{2}{*}{ Campos semánticos } & \multicolumn{2}{|c|}{$5.3-5.5$ años } & \multicolumn{2}{|c|}{5.6 - 5.8 años } & \multicolumn{2}{|c|}{$5.9-5.11$ años } & \multirow{2}{*}{$F$} & \multirow{2}{*}{$P$} \\
\hline & $M$ & $D E$ & $M$ & $D E$ & $M$ & $D E$ & & \\
\hline Vestuario & .48 & .31 & .52 & .32 & .50 & .31 & 1.99 & .136 \\
\hline Animales & .71 & .29 & .74 & .27 & .77 & .27 & 4.93 & .007 \\
\hline Alimentos & .58 & .18 & .63 & .16 & .65 & .17 & 16.57 & .000 \\
\hline Medios de transporte & .46 & .24 & .51 & .24 & .52 & .25 & 5.05 & .007 \\
\hline Muebles y utensilios & .57 & .18 & .62 & .18 & .64 & .17 & 18.73 & .000 \\
\hline Profesiones & .35 & .20 & .39 & .20 & .41 & .21 & 9.96 & .000 \\
\hline Lugares & .34 & .22 & .36 & .21 & .39 & .22 & 5.79 & .003 \\
\hline Formas y colores & .82 & .20 & .86 & .18 & .89 & .15 & 16.43 & .000 \\
\hline Juguetes e instrumentos musicales & .52 & .22 & .56 & .21 & .58 & .22 & 10.48 & .000 \\
\hline
\end{tabular}

$n=1.564$ 


\section{Relaciones semánticas}

En la Tabla 7 se presenta el análisis de las características de las relaciones semánticas de tipo jerarquización de significados en función de la variable sexo, donde no se encontraron diferencias entre varones y mujeres ( $p>.05)$, aunque las medias en cohipónimos e hiperónimos son mayores en las mujeres, y en hipónimos en el caso de la hombres.

Tabla 7

Diferencia de medias en las relaciones semánticas en función del sexo

\begin{tabular}{lcccccc}
\hline \multirow{2}{*}{ Relaciones semánticas } & \multicolumn{2}{c}{ Femenino } & \multicolumn{2}{c}{ Masculino } & \multirow{2}{*}{$t$} & \\
\cline { 2 - 6 } & $M$ & $D E$ & $M$ & $D E$ & 2.46 & 1.15 \\
Hiperónimos & 5.02 & 2.57 & 4.86 & .251 & .37 & .709 \\
Hipónimos & .29 & .57 & .30 & .60 & -.37 \\
Cohipónimos & 13.73 & 5.44 & 13.50 & 5.47 & .78 & .438 \\
\hline$n=1.564$ & & & & &
\end{tabular}

En la Tabla 8 se aprecia que a nivel de hiperónimos y cohipónimos, los grupos de edad se diferencian de forma estadísticamente significativa $(p<.05)$; por ello, para ambos casos se realizó el análisis post hoc con la prueba de Bonferroni.

En los hiperónimos, las diferencias $(p<.01)$ se encuentran entre los grupos de edad de 5 años 3 meses a 5 años 5 meses y de 5 años 6 meses a 5 años 8 meses, siendo los primeros quienes cometen mayores errores de este tipo.
En relación a los cohipónimos, el grupo de edad de 5 años 3 meses a 5 años 5 meses se diferencia significativamente $(p<.01)$ tanto del grupo de 5 años 6 meses a 5 años 8 meses como del grupo de 5 años 9 meses a 5 años 11 meses. En ambos casos, los niños que pertenecen al menor rango de edad son quienes presentan un mayor número de errores de este tipo.

Tabla 8

Análisis de varianza de las relaciones semánticas entre los grupos etários de 5.3 - 5.5 años, 5.6 - 5.8 años y 5.9 - 5.11 años

\begin{tabular}{lccccccccc}
\hline \multirow{2}{*}{ Relaciones semánticas } & \multicolumn{2}{c}{$5.3-5.5$ años } & \multicolumn{2}{c}{$5.6-5.8$ años } & $5.9-5.11$ años & \multirow{2}{*}{$F$} \\
\cline { 2 - 7 } & $M$ & $D E$ & $M$ & $D E$ & $M$ & $D E$ & & \\
\hline Hiperónimo & 5.188 & 2.59 & 4.77 & 2.53 & 4.88 & 2.39 & 3.67 & .026 \\
Hipónimo & .325 & .61 & .29 & .59 & .26 & .53 & 1.24 & .289 \\
Cohipónimo & 14.53 & 5.14 & 13.44 & 5.72 & 12.64 & 5.30 & 13.22 & .000 \\
\hline
\end{tabular}
$n=1.564$

\section{Discusión}

De acuerdo con los objetivos planteados en el estudio a través de los cuales se aborda la semántica desde una perspectiva aplicada (Acosta y Moreno, 2005); es decir, considerando el análisis del léxico y del significado, se sustentarán los resultados sobre la base de tres principales variables de análisis: repertorio léxico, campos semánticos y relaciones semánticas.

Con relación al repertorio léxico, no se encontraron diferencias significativas en función de la variable sexo, de manera similar a lo reportado por Murillo (2009) al evaluar la riqueza léxica en niños de 4 a 6 años en Costa Rica. Por su parte, y contrario a los resultados de este estudio, Dale (1989) y 
Karmiloff y Karmiloff-Smith (2005) sí señalan que existen marcadas diferencias en el tamaño y contenido del léxico infantil entre niños y niñas. Sobre el particular, coincidimos con Huttenlocher (citado por Galián, et al., 2010) quien plantea que las diferencias en función del sexo se observan principalmente en la etapa de adquisición de las primeras 50 palabras, desapareciendo aproximadamente a partir de los 24 meses, etapa a partir de la cual la variable sexo no genera diferencias significativas.

En función de la variable edad sí se encontraron diferencias estadísticamente significativas entre los tres grupos de edad evaluados. Según el análisis post hoc realizado con la prueba Bonferroni, se apreciaron las diferencias entre el grupo de niños de 5 años 3 meses a 5 años 5 meses con los grupos de 5 años 6 meses a 5 años 8 meses y 5 años 9 meses a 5 años 11 meses, correspondiendo el menor rendimiento al grupo de menor edad. En este sentido, se corrobora lo señalado por Galian et al. (2006) quienes indican que existen diferencias individuales en la adquisición del lenguaje inicial, sobre todo en el ritmo y estilo para aprender palabras, lo cual hace pensar que, conforme se incrementa la edad del niño, se incrementa también el repertorio de palabras, lo cual se asocia a mayores posibilidades de interacción y experiencias tanto a nivel familiar, amical y educativo, entre otros.

Respecto de los campos semánticos, se identificaron diferencias significativas, a nivel general, en todos con excepción de vestuario y medios de transporte. Los resultados concuerdan con lo señalado por Torres, Piñeiro, Morenza e Inguanzo (2000) quienes afirman que el desarrollo de las categorías o campos semánticos no es homogéneo en los niños y además, que las diferencias en su desarrollo dependen de su propia naturaleza (generalidad, grado de abstracción, etc.) y de la posibilidad de interacción del niño con los ejemplares. En este sentido, los resultados se pueden explicar señalando que en las categorías vestuario y medios de transporte no se presentan diferencias significativas debido a que son campos con un número de elementos con posibilidades de ampliación bastante reducida y que, además, sus elementos, casi en su totalidad, pertenecen al entorno cercano del niño.

En cuanto a la variable sexo, se encontraron diferencias significativas en el campo semántico vestuario, a favor de las niñas, y en los campos semánticos animales, medio de transporte, lugares, juguetes e instrumentos musicales, a favor de los niños. Estos hallazgos se corroboran con lo señalado por Alva, Hernández-Padilla y Carrión (2005) quienes al evaluar campos semánticos en niños mexicanos de 5 a 12 años identificaron diferencias entre niños y niñas. Del mismo modo, coinciden con lo reportado por Torres et al. (2000) en relación con la diferencia en el desarrollo asociada de la posibilidad de interacción con los elementos del campo semántico. Así, a través del juego y de las experiencias cotidianas es mayor la posibilidad de interacción de las niñas con prendas de vestir y de los niños con animales, medios de transporte, de juguete e instrumentos musicales, principalmente.

En cuanto a la edad se encontraron diferencias significativas en todos los campos semánticos con excepción de vestuario, lo cual probablemente se relacione también con la familiaridad hacia los 
estímulos en los tres grupos de edad evaluados. El análisis post hoc mediante la prueba de Bonferroni evidenció que en los campos animales y lugares solo existen diferencias significativas entre el grupo de 5 años 9 meses a 5 años 11 meses y el grupo de 5 años 3 meses a 5 años 5 meses, con un mejor desempeño en el grupo de mayor edad. Asimismo, en los campos alimentos, transportes, muebles, profesiones y juguetes e instrumentos, además de existir diferencias significativas entre los grupos de edad mencionados, también hubo diferencias entre los grupos de 5 años 6 meses a 5 años 8 meses y el grupo de 5 años 3 meses a 5 años 5 meses, también a favor del primer grupo. En los campos semánticos formas y colores existen diferencias significativas entre cada par de grupo de edad evaluado, siempre a favor de los grupos de mayor rango de edad. Frente a lo mencionado, podemos señalar que pese a la corta diferencia entre cada rango de edad, se observan diferencias significativas con mejores resultados en el grupo de niños de mayor edad.

En cuanto a las relaciones semánticas de tipo jerarquización de significado, a nivel general, se registró un mayor número de cohipónimos seguido de los hiperónimos. Es decir, el error más común de los niños fue verbalizar un término de la misma categoría, por ejemplo decir "naranja" en lugar de "mandarina". En un segundo lugar, las fallas en la precisión léxica estuvieron relacionadas con la denominación de la categoría a la que pertenecía el objeto presentado, por ejemplo, decir "ropa o prenda de vestir" en lugar de "polo" (polera, camiseta).

En cuanto a la variable sexo, no se encontraron diferencias significativas, es decir, los niños y las niñas presentan similar número de hiperónimos, hipónimos y cohipónimos cuando no logran denominar con precisión la imagen presentada.

A nivel de la edad se identificaron diferencias significativas en los hiperónimos y cohipónimos. En los primeros, las diferencias se encuentran entre los grupos de 5 años 3 meses a 5 años 5 meses y de 5 años 6 meses a 5 años 8 meses, siendo los primeros quienes cometen más errores de este tipo. En relación con los cohipónimos, el grupo de edad de 5 años 3 meses a 5 años 5 meses se diferencia significativamente tanto del grupo de 5 años 6 meses a 5 años 8 meses como del grupo de 5 años 9 meses a 5 años 11 meses. En ambos casos, los niños que pertenecen al menor rango de edad son quienes presentan un mayor número de errores de este tipo. Datos que guardan relación con los hallazgos generales en cuanto a la relación semánticas de tipo jerarquización de significado.

Finalmente, podemos concluir señalando que tanto a nivel de repertorio léxico como de campos semánticos y relaciones semánticas, los resultados analizados, considerando la variable edad, muestran diferencias estadísticamente significativas en los tres grupos evaluados (5 años 3 meses a 5 años 5 meses, 5 años 6 meses a 5 años 8 meses y 5 años 9 meses a 5 años 11 meses), pese a que los rangos consideran solo tres meses. En este sentido, el hallazgo se sustenta con lo señalado por Trigo (citado por Ruiz, 2000), quien encontró que en el desarrollo del lenguaje, la variable edad es un factor que genera mayor diferencia que la variable sexo, tal como se corrobora en el presente estudio donde la variable sexo no genera diferencias significativas. 
Como un aporte, tal como señala Rojas (2005), el conocimiento del repertorio léxico, campos semánticos y relaciones semánticas que los niños manejan de manera natural como parte de su desarrollo semántico es de gran importancia para el sistema educativo, ya que deberá ser utilizado por ejemplo en la elaboración del material educativo (textos, guías de trabajo, etc.), así como para la elaboración de propuestas metodológicas diversas.

\section{Referencias}

Acosta, V. y Moreno, A. (2005). Dificultades del lenguaje en ambientes educativos. Del retraso al trastorno específico del lenguaje (1a ed.). Barcelona: Masson.

Acosta, V., Moreno, A., Ramos, V., Quintana, A., y Espino, O. (2002). La evaluación del lenguaje. Teoría y práctica del proceso de evaluación de la conducta lingüística infantil (2a ed.). Málaga: Aljibe.

Alva, A., Hernández-Padilla, E., y Carrión, R. (2005). Habilidades lingüísticas y diferencias de género: análisis de vocabulario. Revista Mexicana de Psicología, 22(1),107120.

Dale, P. (1989). Desarrollo del lenguaje. Un enfoque psicolingüístico. México: Trillas.

Furquim de Andrade, C., Befi-Lopes, D., Dreux, F., y Fiszbein, H. (2004). Teste de linguagem infantil nas áreas de fonologia, vocabulário, fluência e pragmática. São Paulo: Pró-Fono.

Galián, M., Ato, E. y Carranza, J. (2010). Explosión del vocabulario infantil y primer lenguaje: una revisión. Anales de Psicología, 26(2), 341-347.
Galián, M., Carranza, J., y Escudero, A. (2006). Variación estilística en la adquisición del léxico inicial. Anales de Psicología, 22(1), 98-104.

Gallego, J. (2006). Enciclopedia temática de logopedia. Málaga: Aljibe.

Hernández, S., Fernández, C., y Baptista, L. (2006). Metodología de la investigación. México, DF: McGraw-Hill.

Karmiloff, K. y Karmiloff-Smith, A. (2005). Hacia el lenguaje. Madrid: Ediciones Morata.

Martínez, E. (2002). Lingüística. Teoría y aplicaciones. Barcelona: Masson.

Mayor, M., Zubiauz, B., y Díez-Villoria, E. (2005). Estudios sobre la adquisición del lenguaje. Salamanca: Ediciones Universidad de Salamanca.

Moreno, N. (2008). Lingüística general II: Semántica Léxica. UCLM-CEU Talavera. http://www.quiben.org/32030/wpcontent/uploads/2008/01/apuntes-semantica.pdf

Murillo, M. (2009). Diversidad de vocabulario en los preescolares aportes para valorar su competencia léxica. Filología y Lingüística, 35(1), 123-138.

Navarro, A. (2006). Relaciones semánticas entre las palabras: hiponimia, sinonimia, polisemia, homonimia y antonimia. Los cambios semánticos. Aldadis.net La revista de educación, 10(3). Recuperado de http://www.aldadis.net/revista10/documentos/22.pdf

Rojas, M. (2011). Una responsabilidad escolar olvidada: el desarrollo del componente léxico. Revista Educación, 29(1), 31-44.

Ruiz, M. (2000). Cómo analizar la expresión oral de los niños y niñas. Málaga: Aljibe.

Torres, R., Piñeiro, A., Morenza, L., e Inguanzo, G. (2000). Estudio de seis categorías semánticas en niños pequeños. Revista Cubana de Psicología, 17(2), 106-113. 